\title{
The usefulness of Duplex Doppler ultrasound in the angiological and dermatological diagnosis of patients with blue toe syndrome
}

\author{
Katarzyna Pawlaczyk¹, Marcin Gabriel², Daria A. Strzelecka-Węklar³, Zbigniew Krasiński², Michal Stanisic ${ }^{2}$, \\ Zofia Gabriel $^{4}$, tukasz Dzieciuchowicz ${ }^{3}$, Zygmunt Adamski ${ }^{3}$ \\ ${ }^{1}$ Department of Hypertensiology, Angiology and Internal Diseases, Poznan University of Medical Sciences, Poznan, Poland \\ 2Department of General and Vascular Surgery, Poznan University of Medical Sciences, Poznan, Poland \\ ${ }^{3}$ Department of Dermatology, Poznan University of Medical Sciences, Poznan, Poland \\ ${ }^{4}$ Hospital of Neuropsychiatry, Kościan, Poland \\ Adv Dermatol Allergol 2017; XXXIV (5): 478-484 \\ DOI: https://doi.org/10.5114/ada.2017.71117
}

\begin{abstract}
Introduction: Peripheral microembolism is one of the most frequent causes of acute limb ischemia. In order to effectively prevent relapses it is essential to localize and eliminate the source of embolism.

Aim: To evaluate the role of Duplex Doppler ultrasound examination in identifying the causes of blue toe syndrome (BTS).

Material and methods: The group of 165 patients with clinical symptoms of BTS on their upper limbs $(n=16)$ and lower limbs $(n=149)$ was investigated. They all underwent Duplex Doppler ultrasound of the major arteries of the extremities, where ischemic changes occurred.

Results: Morphological and functional changes which might be potential sources of microembolism were identified in 146 patients. These changes included significant short-length stenoses or unstable atherosclerotic plaque $(n=73)$, true aneurysms $(n=42)$ and pseudoaneurysms $(n=17)$. In 11 cases, pathology of vascular prostheses in the form of anastomotic aneurysms, infection and residual thrombi after fibrinolysis was detected. In all cases, Duplex diagnosis was confirmed by other imaging and intraoperative tests.

Conclusions: Duplex Doppler ultrasound of the arteries in the affected limb with a full length view should be the first-line examination in diagnosing patients with BTS. In the absence of hemodynamic blood flow disturbances in the major arteries in patients with symptoms of BTS, it is advisable to start haematological tests to identify/exclude congenital or acquired thrombophilia.
\end{abstract}

Key words: blue toe syndrome, Duplex examination, peripheral embolism.

\section{Introduction}

The term "blue toe syndrome" (BTS) was first used by Komody in his publication dated 1976. It was described as "acute pain of one or more toes, accompanied by cyanosis, and of vascular aetiology confirmed by angiography" [1]. Another definition says that BTS is a blue or purple discoloration of one or more toes in patients with a proven history of no injuries, no thermal trauma caused by low temperature and with no systemic symptoms of cyanosis caused by methemoglobinemia or hypoxia. Typical skin symptoms include cyanosis, livedo reticularis, purpura, ulcerations and even necrosis. Patients com- plain of irritating acute pain [2]. Similar symptoms are typical for thrombosis in the course of antiphospholipid syndrome, cancer, thrombocytopenic purpura, disseminated intravascular coagulation and warfarin-induced necrosis. They can also be the effect of acrocyanosis, chilblains, chilblain lupus erythematosus, the action of vasoconstrictors and inflammations of infective origin, such as in the course of syphilis and purulent infections, and non-infective origin, such as Behçet's disease, various forms of vasculitis, calciphylaxis and venous outflow disturbances in the course of venous thrombosis. Symptoms similar to BTS develop also in diseases leading to disorders of the blood constituents: paraproteinemia,

Address for correspondence: Katarzyna Pawlaczyk, Department of Hypertensiology, Angiology and Internal Diseases, Poznan University of Medical Sciences, 1/2 Długa St, 61-848 Poznan, Poland, phone: +48 600393 814, e-mail: kati2911@poczta.onet.eu Received: 23.02.2016, accepted: 27.06.2016. 
myeloproliferative disorders, polycythemia vera, thrombocytopenia, cryofibrinogenemia, cryoglobulinemia, cold agglutinin diseases [2].

Peripheral microembolism is one of the most frequent causes of the development of BTS symptoms. Embolic material includes microthrombi, cholesterol crystals or fibrinplatelet aggregates. Embolic events are often caused by the atherosclerotic plaque located within aorta and vessels in the lower limbs or thrombi within the lumen of the aneurysm [3]. Due to the significant role of skin symptoms, patients usually see the dermatologist first. One must remember that despite vascular causes of the disease, clinical examination often shows pulse on peripheral arteries, which may distract doctor's attention from the correct diagnosis. It must be also noted that in cases of the identified source of embolism, an immediate and effective vascular surgery may resolve the skin symptoms without the need for dermatological treatment. Therefore, quick and correct diagnosis is so important in the treatment of BST. It helps to find sources of embolism and exclude other causes of symptoms similar to BST, which normally do not require such urgent intervention. Correctly performed Duplex examination seems particularly useful in this case, both for making a correct diagnosis and in numerous cases for selecting the final form of treatment [4].

\section{Aim}

The aim of this study was to evaluate the role of Duplex Doppler ultrasound examination in identifying the causes of BTS.

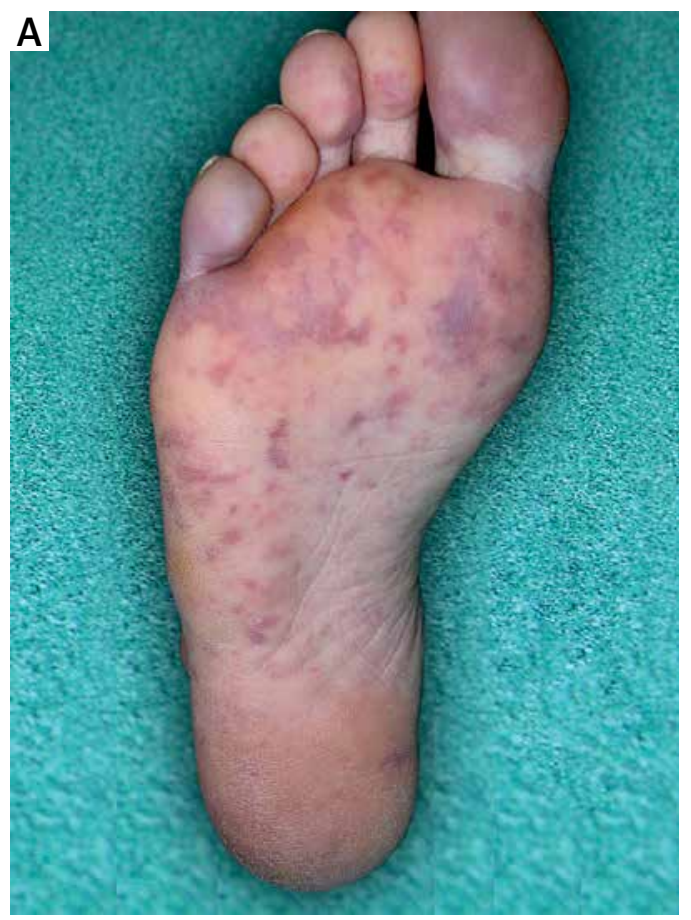

\section{Material and methods}

The study group consisted of 165 patients who were diagnosed and treated in 2004-2015 for the symptoms of BTS in the Department of General and Vascular Surgery and its outpatient unit, and the Department of Hypertensiology, Angiology and Internal Medicine of the Medical University in Poznan. The study group included 91 men and 39 women at the age of $17-88$ years (average age: 56.9).

In the period between 1 and 6 months prior to the study, all patients developed focal lesions on the tips of their toes and/or on the plantar surface of the foot $(n=149$, Figure 1$)$ or on their fingertips ( $n=16$, Figure 2). These lesions were accompanied by irritating pain or burning sensation in the affected area. In 139 patients, the number of focus points remained unchanged while in 26 patients the number increased during the course of the disease.

In all patients, physical examination demonstrated correct or slightly altered temperature in the affected limbs with correct vascular peristalsis on the tips of the toes without any lesions. In 67 patients, the examination revealed absent pulse in popliteal fossa and/or feet while in 2 patients with lesions on their hands, positive Adson's and Patrick's tests were observed.

All patients had a history of acute ischemic episodes of category I according to the clinical classification by SVS/ISCVS.

Comorbidities are presented in Table 1. Eighty percent of the patients received one or two antiplatelet drugs (acetylsalicylic acid at a dose of 75-150 mg/day or clopidogrel $1 \times 75 \mathrm{mg} /$ day). $10.3 \%$ of patients received

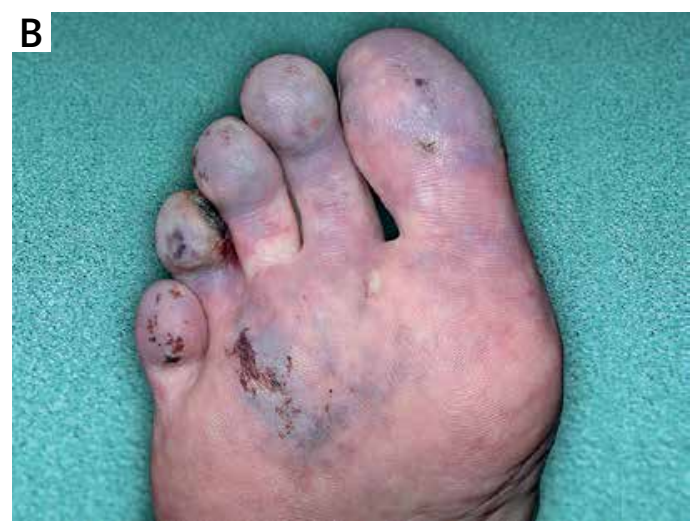

Figure 1 A, B. Skin lesions affecting the foot in the course of BTS 


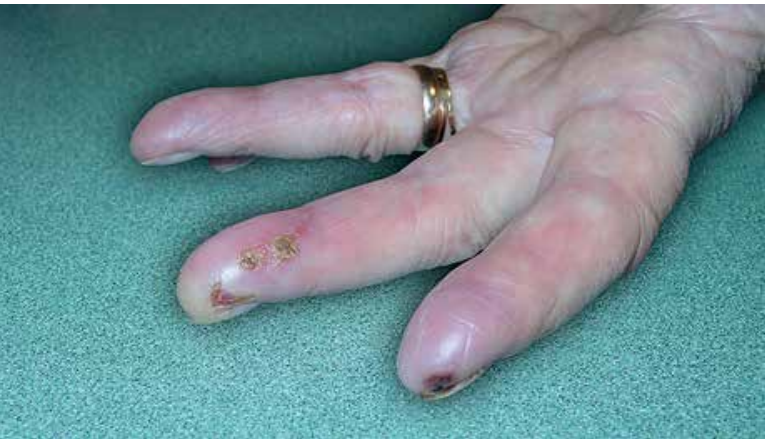

Figure 2. Skin lesions affecting fingers in the course of BTS

coumarin derivatives due to the permanent atrial fibrillation. $73.3 \%$ received statins.

In all patients, antithrombotic treatment in the form of low-molecular-weight heparins at a therapeutic dose, i.e. nadroparin $0.1 \mathrm{ml} / 10 \mathrm{~kg} /$ day or dalteparin at a dose of $200 \mathrm{U} / \mathrm{kg} /$ day was introduced on an ad hoc basis. All patients had Duplex Doppler examination of aorta and limb arteries performed. The procedures were done using Logic 7 and Mindray DC-8 ultrasound with 3.5-4.5 MHz curved transducers and 5-12 MHz linear transducers. The examinations were performed by two highly experienced vascular surgeons (each had performed at least 50,000 examinations of peripheral arteries) according to the recommendations of the Polish Society for Vascular Surgery [5]. In most patients, preliminary Duplex Doppler diagnosis was verified by further imaging tests - Angio-CT $(n=57)$ or DSA during endovascular interventions $(n=91)$.

Patients with no morphological sources of microembolism identified in imaging underwent hematologic tests (for congenital or acquired thrombophilia) and cardiologic tests (for cardiac arrhythmias and valve diseases).

\section{Results}

Morphological vascular changes which might be the potential sources of peripheral embolism were recog-
Table 1. General characteristics of the patients

\begin{tabular}{lc}
\hline Parameter & Value \\
\hline Age [years] (range/average) & $17-88 / 56.9$ \\
\hline Women/men & $55 / 91$ \\
\hline Smokers & $99(67.8 \%)$ \\
\hline Hyperlipidaemia & $74(50.6 \%)$ \\
\hline Diabetes & $23(15.7 \%)$ \\
\hline Hypertension & $89(60.9 \%)$ \\
\hline
\end{tabular}

nized by Duplex Doppler examination in 146 patients. The location and nature of changes in specific parts of the vessels are presented in Table 2.

In all patients with BTS symptoms on their upper limbs, changes in subclavian arteries (SA) were identified. Hemodynamically significant stenosis of proximal subclavian arteries was identified in 10 patients with alteration of the blood flow spectrum in homonymous vertebral arteries (steal syndrome type I, grade I or II). In one patient without any clinical or ultrasound symptoms of the steal syndrome, stenosis of the middle segment of subclavian artery was identified (Figure 3). The presence of stenoses was confirmed during an angioplasty surgery of the narrowed vessels. Genuine aneurysms of the proximal SA and two iatrogenic pseudoaneurysms in the middle segment of SA were treated endovascularly by implantation of coated stents (Figure 4).

The presence of compression symptoms within thoracic inlet was identified during evaluation of the flow in the veins and axillary arteries with functions tests. The diagnosis was confirmed by angio-CT performed in abduction. One patient had a first rib removed and another patient had a corrective surgery of poorly-healed clavicle fracture.

With regards to lower limbs, sources of microembolism were dominated by atherosclerotic lesions $(n=68)$ in the form of short-length significant stenoses $(n=52)$ (Figure 5), unstable atherosclerotic plaque $(n=5)$, ob-

Table 2. Morphological lesions detected by Duplex examination of the main arteries in the limbs, which might be the potential source of microembolism

\begin{tabular}{|c|c|c|c|c|c|c|}
\hline \multirow[t]{2}{*}{ Vessel } & \multicolumn{2}{|c|}{ Aneurysms } & \multirow[t]{2}{*}{ Stenosis } & \multirow[t]{2}{*}{ Obstruction } & \multirow[t]{2}{*}{ Other } & \multirow[t]{2}{*}{ Total } \\
\hline & True & False & & & & \\
\hline Aorta & 16 & & 2 & & & 18 \\
\hline Iliac arteries & 7 & & 21 & 1 & & 29 \\
\hline Superficial femoral artery & 17 & 9 & 29 & 4 & $5^{1}$ & 64 \\
\hline Popliteal artery & 1 & 2 & 5 & & & 8 \\
\hline Prostheses & & $4^{2}$ & $5^{3}$ & & $2^{4}$ & 11 \\
\hline Subclavian arteries & 1 & 2 & 11 & & $2^{5}$ & 16 \\
\hline Total & 42 & 17 & 73 & 5 & 9 & 146 \\
\hline
\end{tabular}

${ }^{1}$ Stent restenosis, ${ }^{2}$ anastomotic aneurysms, ${ }^{3}$ the presence of residual mural thrombi in prostheses open with fibrinolysis, ${ }^{4}$ prosthesis infection with fluid sections around the body of the prosthesis, ${ }^{5}$ thoracic inlet syndrome. 
struction in the course of arterial thrombosis $(n=5)$ or hemodynamically significant restenoses in the lumen of previously implanted stents $(n=5)$. In the vast majority of cases, the above-listed lesions were short-length.

The second most frequent sources of microembolism were genuine aneurysms $(n=41)$ within the distal abdominal aorta $(n=16)$, various segments of superficial femoral arteries $(n=17)$ or popliteal artery $(n=1)$. Stratified, mural, partly free-floating thrombi were always observed in aortic and iliac artery aneurysms (Figure 6). However, when it comes to the segments of superficial femoral arteries affected by aneurysmal changes, the thrombi were present only in 10 out of 17 cases. In the remaining patients, microembolism was caused by turbulences in the narrowed or dilated vascular segments.

Fifteen pseudoaneurysms included 8 iatrogenic complications following endovascular procedures involving puncture of the common and superficial femoral arteries, one complication following rupture of genuine aneurysm in the distal SFA and 2 complications of posterior knee dislocation accompanied by popliteal artery contusion.

In 11 patients with previously implanted Dacron vascular grafts, the sources of peripheral microembolism included anastomotic aneurysms developed within endto-side anastomosis in the groin $(n=4)$, residual thrombi in the lumen of prostheses treated by topical fibrinolysis due to thrombosis $(n=5)$ and mural thrombi developed in the lumen of prostheses surrounded by thin fluid space and suspected of infection $(n=2)$.

In 19 patients with BTS, no macroscopic vascular lesions were identified by Duplex examination. Additional tests revealed thrombocythemia in the course of the neoplastic process or following the removal of the spleen in 2 patients and various forms of thrombophilia in

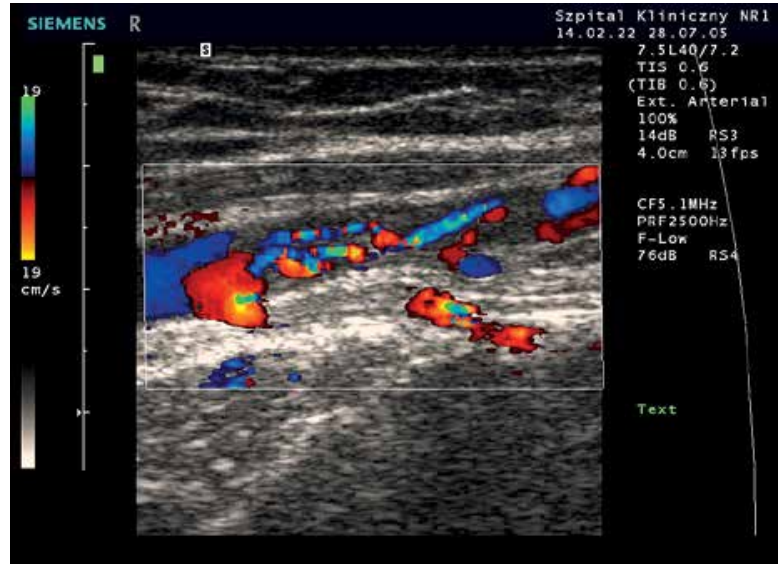

Figure 3. Segmental stenosis of the middle segment of the subclavian artery caused by previous embolism

11 patients. In 3 patients, who developed the symptoms of BTS in the early postoperative period after implantation of aorto-bi-iliac prostheses due to abdominal aortic aneurysm or aortobifemoral prostheses due to the aorticfemoral obstruction, haematological tests revealed disseminated intravascular coagulation (DIC) that required long-term treatment with anticoagulants.

Only in 3 patients no cause was identified. However, the introduction of anticoagulants resolved the symptoms.

Preliminary diagnosis made on the basis of Duplex Doppler examination was confirmed in $98.6 \%$ by other imaging tests. The failure in 3 cases was caused by the difficulties in unambiguous evaluation of the iliac arteries, which visualization was obscured by intestinal gases. In 121 patients surgeries were performed. The type and scope of the surgery was dependent on the nature of the identified vascular lesions. In patients with hematologic
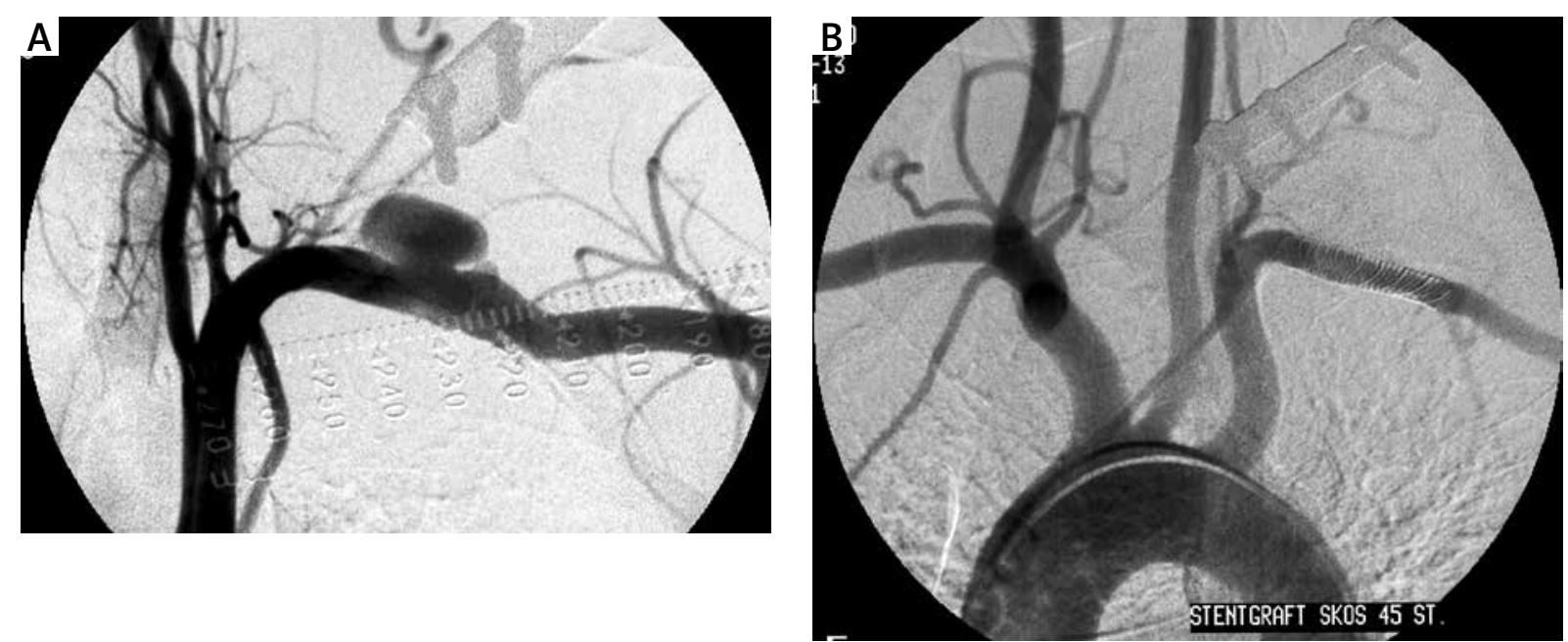

Figure 4. Angiography of the subclavian artery: A - pseudoaneurysm following iatrogenic arterial injury; B - status post coated stent placement 

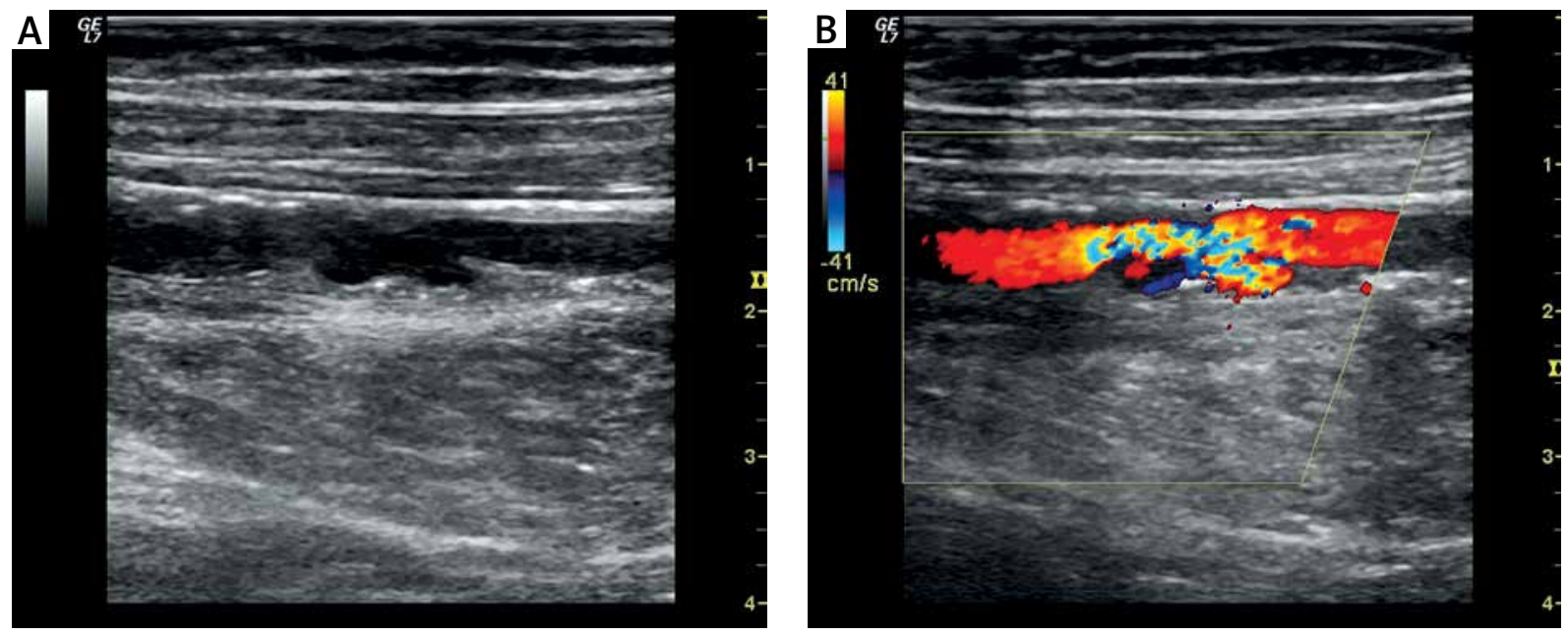

Figure 5 A, B. Short-length significant stenosis of the superficial femoral artery

disorders, adequate antiplatelet agents and anticoagulants were introduced, depending on the aetiology.

A complete halt of the development of new ischemic focus points and the gradual healing of previously developed lesions was achieved in 157 patients. Six and 2 patients had toes and forefeet amputated, respectively. Minor amputations were carried out in 6 patients with antiphospholipid syndrome and one patient after infected prosthesis replacement.

\section{Discussion}

Performing an adequate imaging test in patients with BTS is so important because in most cases, the disease is not manifested by an abnormal pulse rate or anklebrachial index, which may encourage the physicians to exclude vascular aetiology.

All previous reports on blue toe syndrome showed that BTS affected lower limbs more often than upper limbs [6-

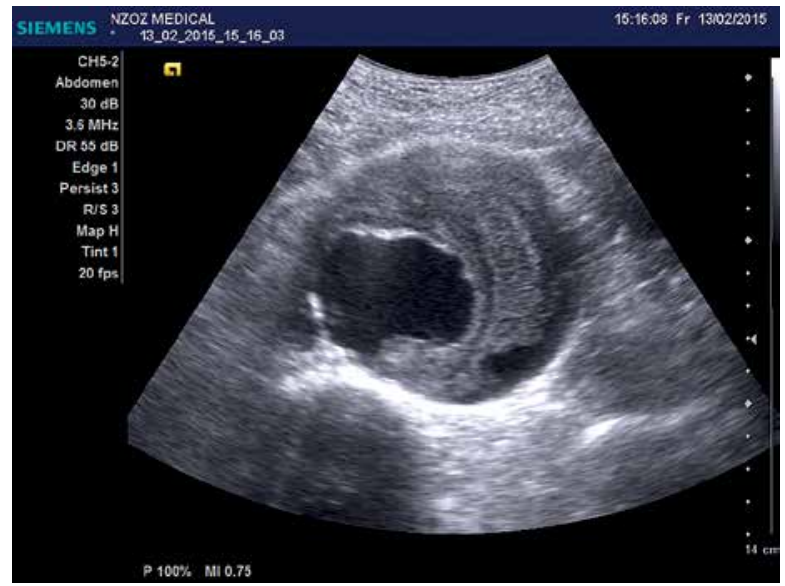

Figure 6. Free-floating thrombus in the lumen of a true aneurysm of the abdominal aorta
10]. In our patients, lesions located on the feet dominated likewise. Focal lesions on hands were observed only in 16 patients; in all cases, the sources of microembolism in the form of morphological changes in subclavian vessels were identified. Although Spittel claimed that the only cause of microembolism in the upper limb was subclavian arteries stenosis, our patients developed other changes in the form of aneurysms and compression within thoracic inlet [8]. It seems that frequent identification of atherosclerotic changes may be the effect of the possibility for interim evaluation, for instance on the basis of finding subclavian artery steal syndrome. The presence of significant subclavian artery stenosis is usually connected with II grade steal syndrome, i.e. the presence of bidirectional flow in the vertebral artery. Rare detection of other vascular causes of BTS within upper limbs may be the effect of difficulties in their visualization in Duplex Doppler examination. The detection of true aneurysms requires mastering the technique of examination of fossa jugularis with a phased array or curved transducers [5, 11]. In turn, the confirmation of the thoracic inlet syndrome requires knowledge of specific function tests to confirm the presence of compression.

In case of lower limbs we have more diversified causes of microembolism in terms of mechanism and location. The most common sources are atherosclerotic plaque and true aneurysms. Spittel claims that thrombi may develop not only in ulcerations, but also in single 2-4 $\mathrm{mm}$ thick debris of irregular shape. There is lack of clarity in previous studies regarding the dominant location of the atherosclerotic plaque causing embolism. According to Komody, the above-mentioned changes are located mostly in femoropopliteal segments [1]. More recent studies by Katz and Spittel, in turn, show the growing share of location in the iliac arterial segments $[6,8]$. It is believed that the shift in paradigms may be the effect of the growing frequency of performing Duplex Doppler and echocardiography to identify changes which 
might be the potential sources of microembolisms [7, 10, 12]. Katz claims that the real-time tests allow for visualization of free-floating thrombi in the chamber of aneurysms or adhered to the debris [6]. Their detection is not possible by analysing still images obtained in other tests such as DSA, angio-CT or MRT. The advantage of Duplex Doppler examination in detection of short-term and mobile changes over other imaging tests has been shown by previous studies [4, 13]. Additionally, angiography, which does not allow to identify ulcerated and small mural deposits, increases the risk of various forms of embolism during instrumental manipulation in large vessels [10]. Even repeated DSA in various projections is of limited use. Similar observations were made by radiologists in relation to ultrasound examinations performed by technicians. The evaluation of still images prevented the detection of free-floating thrombi visualized only during examination performed by the physicians [8].

Another, next to Duplex test, highly sensitive method of detecting even small free-floating thrombi in the descending aorta is transoesophageal echocardiography [7, $9,12]$. It allowed for the detection of 4 times more potential sources of microembolism than the transthoracic examination [6].

The location of lesions in the abdominal aorta contributes to the development of lesions in both limbs. In Katz's studies bilateral lesions were present in 14 out of 47 patients and they were all due to the lesions located in the aorta. In case of unilateral location of ischemic changes, the presence of potential sources of embolism in the aorta were detected only in 3 out of 33 patients. In the remaining cases, potential sources were located in the main arteries of specific limbs [6]. Previous studies drew attention to the possibility of the presence of true aneurysms and significant stenoses of femoral, iliac and popliteal arteries. In our patients, the atherosclerotic plaque was dominated by single, short-length, significant stenoses located in femoral or superficial femoral arteries. Apart from the above-listed sources of microembolism, in our patients we also detected the presence of pseudoaneurysms, traumatic and iatrogenic aneurysms and residual thrombi in thrombosed and anti-thrombolitically treated vascular prostheses. In most cases, the introduction of fibrinolysis allows for partial restoration of the patency of the thrombosed vessel while the remaining thrombi are weakly adhered to the wall of the vessels or prostheses.

The development of microthrombi on small atherosclerotic changes of the aorta and peripheral arteries is facilitated by the coexistence of coagulation disorders in the form of myeloproliferative diseases, neoplastic processes, hyperfibrinogenaemia and hypercysteinemia [6, $14,15]$. Myeloproliferative diseases result from enhanced platelet aggregation. Similarly, in 50\% of cancerous patients and $95 \%$ of patients with metastases, enhanced platelet aggregation and affinity for thrombin and ad- enosine phosphate were observed. In $60 \%$ of cancerous patients, the number of thrombocytes increases [16, 17].

Detection of the morphological lesion which might be the potential source of microembolism is an indication for surgical treatment, widely recognized as the basic form of treatment in patients with BTS. It allows for the preservation of $90 \%$ of limbs and reduction of the number of amputations to 5\%. The proposed surgical procedures include a wide spectrum of vascular surgical techniques: unblocking, segmental ligation, excision of the affected segment, with a consequent by-pass procedure. The type of the procedure depends upon the nature of the location and extent of the primary lesion $[10,18]$. In recent years endovascular procedures have been more often recommended as an alternative procedure for open surgeries. However, some authors point to the increased risk of embolic episodes as a result of intravascular manipulation during angioplasty and stent placement [19, 20]. Due to the fact that the release of microemboli is most often observed during preliminary balloon angioplasty, it is recommended to use self-expandable stents with consequent balloon valvuloplasty in patients with BTS. Such procedure, according to Zhang, limits the significant risk of secondary embolic complications [9].

In case of accompanied morphological vascular lesions and homeostatic disorders, it is recommended to introduce haematological therapy prior to the angioplasty and stent placement [6]. Such procedure limits the risk of developing symptoms of microembolism during endovascular surgeries.

We usually treated pseudoaneurysms of our patients by injecting thrombin into the chamber. In all cases, the treatment proved effective, with no adverse events. To prevent surgical complications by peripheral embolism one must observe specific recommendations, i.e. performing only ultrasound-guided puncture of the lesion and administering thrombin in volume several times smaller than the volume of the treated chamber [13].

It is generally acknowledged that antithrombotic therapy with heparin or oral medications is of limited efficacy in the reduction of microembolism. Repeated embolic episodes are frequently observed despite adequate antithrombotic therapy $[10,18]$. Nonetheless, it may significantly reduce and shorten the duration of clinical symptoms, which we successfully took advantage of in our patients. Antiaggregants seem effective in inhibiting aggregation and development of new embolic events, however they are ineffective in cholesterol embolism [21].

\section{Conclusions}

The most frequent causes of BTS are aneurysms and short-length significant arterial stenoses. Duplex Doppler ultrasound of the arteries in the affected limb with a full length view, and in case of lower limbs, also the aorta and femoral arteries, should be a first-line diagnostic 
examination. This examination allows for detection of sources of embolism in more than $90 \%$ of patients.

In the absence of hemodynamic blood flow disturbances in the major arteries in patients with symptoms of BTS, it is necessary to start haematological tests to identify/exclude congenital or acquired thrombophilia.

Due to a wide spectrum of skin symptoms in BTS, the dermatologist may play an important role as a person initiating the correct diagnostic process.

\section{Conflict of interest}

The authors declare no conflict of interest.

\section{References}

1. Karmody AM, Powers SR, Monaco VJ, Leather RP. Blue toe' syndrome. An indication for limb salvage surgery. Arch Surg 1976; 111: 1263-8.

2. Hirschmann JV, Raugi GJ. Blue (or purple) toe syndrome. J Am Acad Dermatol 2009; 60: 1-20.

3. Quinones A, Saric M. The cholesterol emboli syndrome in atherosclerosis. Curr Atheroscler Rep 2013; 15: 315.

4. Gabriel M, Pawlaczyk K, Szajkowski R, et al. The use of duplex ultrasound arterial mapping (DUAM) and preoperative diagnostics in patients with atherosclerotic ischaemia of lower extremities. Pol Prz Chir 2012; 84: 486-99 [Polish].

5. Gabriel M, Urbanek T, Madycki G, et al. Duplex Doppler ultrasound examination of extremities arteries: guidelines of the Polish Society for Vascular Surgery. Kard Pol 2014; 72: 662-79 [Polish].

6. Katz SG, Kohl RD. Spontaneous peripheral arterial microembolization. Ann Vasc Surg 1992; 6: 334-7.

7. Roth M, SchoÈnburg M, Kloevekorn WP, Bauer EP. Thrombotic formations within the aortic arch as source of embolization in patients with coagulopathia. Eur J Cardiothoracic Surg 2001; 19: 534-6.

8. Spittell PC, Seward JB, Hailett Jr JW. Mobile thrombi in the abdominal aorta in cases of lower extremity embolic arterial occlusion: value of extended transthoracic echocardiography. Am Heart J 2000; 139: 241-4.

9. Zhang WW, Abou-Zamzam AM, Hashisho M, et al. Characteristics of peripheral microembolization during iliac stenting: Doppler ultrasound monitoring. Eur J Vasc Endovasc Surg 2005; 30: 311-4.

10. Zhang WW, Abou-Zamzam AM, Hashisho M, et al. Staged endovascular stent grafts for concurrent mobile/ulcerated thrombi of thoracic and abdominal aorta causing recurrent spontaneous distal embolization. J Vasc Surg 2008; 47: 193-6.

11. Gabriel M, Pawlaczyk K. Zespół podkradania tętnicy kręgowej - pułapki diagnostyczne. Przew Lek 2004; 7: 102-7 [Polish].

12. Lee JS, Chandraratna PAN. Peripheral embolism from an aortic-arch atheroma. N Engl J Med 2003; 349: e24.

13. Pawlaczyk K, Gabriel M, Juszkat J, et al. Ultrasound assessment of the morphology of iatrogenic pseudoaneurysms as a prognostic factor in compression therapy. Pol I Radiol 2008; 73: 16-21 [Polish].

14. Antecol DH, Walley VM, Chan KL. Massive acute thrombosis of the descending thoracic aorta in heparin-associated thrombocytopenia and thrombosis. I Am Soc Echocardiogr 1994; 7: 550-2.
15. Tunick PA, Lackner H, Katz ES, et al. Multiple emboli from a large aortic arch thrombus in a patient with thrombotic diathesis. Am Heart J 1992; 124: 239-41.

16. Colman RW, Rubin RN. Disseminated intravascular coagulation due to malignancy. Semin Oncol 1990; 17: 172-86.

17. Ey FS, Goodnight SH. Bleeding disorders in cancer. Semin Oncol 1990; 17: 187-97.

18. Lauvao LS, Goshima KR, Leon Jr LR, et al. Superficial femoral artery thrombosis as a cause for distal embolism in primary antiphospholipid syndrome. J Vasc Surg 2008; 48: 472-7.

19. Manninen HI, Rasanen HT, Vanninen RL, et al. Stent placement versus percutaneous transluminal angioplasty of human carotid arteries in cadavers in situ: distal embolization and findings at intravascular US, MR imaging, and histopathologic analysis. Radiology 1999; 212: 483-92.

20. Orlandi G, Fanucchi S, Fioretti C, et al. Characteristics of cerebral microembolism during carotid stenting and angioplasty alone. Arch Neurol 2001; 58: 1410-3.

21. Bruns FJ, Segel DP, Adler S. Control of cholesterol embolization by discontinuation of anticoagulant therapy. Am J Med Soc 1978; 275: 105-7. 